\title{
CARACTERIZAÇÃO DOS PACIENTES COM TRANSTORNO OBSESSIVO COMPULSIVO: UM ESTUDO EPIDEMIOLÓGICO*
}

Adriana Gonçalves de Oliveira ${ }^{1}$, Juliana Ferreira da Silva ${ }^{1}$, Laura Campos Teles ${ }^{1}$, Richardson Miranda Machado ${ }^{2}$

\begin{abstract}
RESUMO: Trata-se de estudo retrospectivo e exploratório, realizado em instituição psiquiátrica com o objetivo de caracterizar os pacientes acometidos por Transtorno Obsessivo Compulsivo quanto ao sexo, idade, proveniência, tempo de internação, tipo de alta, tipo de internação e fonte financiadora da internação. A amostra foi constituída por 61 pacientes sendo predominante o sexo feminino (47-77\%), a faixa etária acima de 41 anos (41-67\%), provenientes da própria família (31-50,8\%) e o tempo de internação de 30 dias (33-54,1\%). A alta médica foi a mais frequente (51-83,6\%), houve prevalências similares entre as primeiras internações (30-49,2\%) e reinternações (31-50,8\%); o principal financiador das internações hospitalares foi o Sistema Único de Saúde (54-88,5\%). Ações que visem diagnosticar precocemente o transtorno devem ser desenvolvidas para que o tratamento seja prontamente iniciado a fim de evitar o agravamento de obsessões e compulsões. PALAVRAS-CHAVE: Transtorno obsessivo-compulsivo; Hospitalização; Enfermagem psiquiátrica.

\section{CHARACTERIZATION OF PATIENTS WITH OBSESSIVE-COMPULSIVE DISORDER: AN EPIDEMIOLOGICAL STUDY}

ABSTRACT: This is a retrospective, exploratory study, undertaken in a psychiatric institution with the aim of characterizing the patients affected by Obsessive-Compulsive Disorder by sex, age, provenance, duration of hospitalization, type of discharge, type of hospitalization, and source of financing of hospitalization. The sample comprised 61 patients, the majority of whom were female (47-77\%), age range over 41 years old (41-67\%), who came to hospital from the family home (31-50.8\%) and length of hospitalization of 30 days (33-54.1\%). The most frequent type of discharge was medical (51-83.6\%), and there were similar prevalences for first hospitalizations (30-49.2\%) and re-hospitalizations (31-50.8\%); the main financer for hospitalization was the Unified Health System (54-88.5\%). Actions for the early diagnosis of this disorder must be developed so that the treatment may be initiated promptly, so as to avoid the worsening of obsessions and compulsions.

KEYWORDS: Obsessive-compulsive disorder; Hospitalization; Psychiatric nursing.

\section{CARACTERIZACIÓN DE LOS PACIENTES CON TRASTORNO OBSESIVO COMPULSIVO: UN ESTUDIO EPIDEMIOLÓGICO}

RESUMEN: Este es un estudio retrospectivo y exploratorio, realizado en institución psiquiátrica con el objetivo de caracterizar los pacientes víctimas del Trastorno Obsesivo Compulsivo cuanto al sexo, edad, proveniencia, tiempo de internación, tipo de alta, tipo de internación y fuente financiadora de la internación. La muestra fue constituida de 61 pacientes, siendo predominante el sexo femenino (47-77\%), la franja etaria de mayores de 41 años (41-67\%), provenientes de la propia familia (31-50,8\%) y el tiempo de internación de 30 días (33-54,1\%). La alta médica fue la más frecuente (51-83,6\%), huvo prevalencias similares entre las primeras internaciones (30-49,2\%) y reinternaciones (31-50,8\%); el principal financiador de las internaciones hospitalares fue el Sistema Único de Salud (54-88,5\%). Es necesario desarrolar acciones cuya finalidad sea diagnosticar de modo precoz el trastorno para que el tratamiento sea luego iniciado a fin de evitar que enpeoren las obsesiones y compulsiones.

PALABRAS CLAVE: Trastorno obsesivo-compulsivo; Hospitalización; Enfermería psiquiátrica.

*Artigo produzido durante a disciplina optativa "Pesquisa em Saúde" do Curso de Graduação em Enfermagem da Universidade Federal de São João Del-Rei - UFSJ.

${ }^{1}$ Acadêmica do Curso de Graduação em Enfermagem da UFSJ.

${ }^{2}$ Enfermeiro. Doutor em Psiquiatria. Professor de Psiquiatria e Coordenador da Disciplina Optativa "Pesquisa em Saúde" do Curso de Graduação em Enfermagem da UFSJ. 


\section{INTRODUÇÃO}

O Transtorno Obsessivo Compulsivo (TOC) é um quadro psiquiátrico caracterizado pela presença de obsessões e compulsões. Obsessões são ideias, pensamentos, imagens ou impulsos repetitivos e persistentes vivenciados como intrusivos e provocam ansiedade. Compulsões são comportamentos repetitivos ou atos mentais que visam reduzir essa ansiedade $^{(1)}$. As obsessões e compulsões não são características exclusivas deste transtorno, podendo ser verificadas também em depressões e esquizofrenia. Nos portadores de TOC, elas formam um círculo vicioso difícil de ser interrompido, uma vez que as compulsões são uma forma de alívio da ansiedade e da aflição causadas pelas obsessões ${ }^{(2)}$.

As obsessões mais comuns são: preocupação com sujeira ou secreções corporais, medo de que algo terrível possa acontecer a si mesmo ou a alguém querido, preocupação com simetria e escrupulosidade. As principais compulsões são: lavagem de mãos, verificação de portas, ordenação e arrumação, contagem e colecionismo ${ }^{(1)}$. É comum aos portadores desse transtorno a presença de temores exagerados, que os leva a evitar tocar em objetos, como móveis, dinheiro e corrimões de escadas e, até mesmo, faz com que eles deixem de frequentar lugares considerados sujos ou contaminados ${ }^{(3)}$.

O TOC é o quarto transtorno mental mais frequente no mundo ${ }^{(4-5)}$. Nos Estados Unidos da América caracteriza-se como o quarto diagnóstico psiquiátrico mais comum após as fobias, abuso de drogas e depressão $0^{(3)}$. Inicia-se, geralmente, no final da adolescência e início da vida adulta, com semelhante distribuição entre os sexos, sendo discretamente maior em mulheres e com preponderância em crianças do sexo masculino.

No mundo, o TOC atinge de $2 \%$ a $3 \%$ da população, o que significa 50 milhões de portadores, com prevalência anual de $1,5 \%$. As taxas do TOC na infância e adolescência se assemelham às dos adultos, variando de $1,9 \%$ a $4 \%{ }^{(6)}$. No Brasil calcula-se que em torno de $2,5 \%$ da população, ou seja, cerca de uma em cada 40 pessoas são portadores de TOC, e destas, $10 \%$ ficam gravemente incapacitados pela doença, pois ela tende a ser crônica ${ }^{(5)}$.

Os sintomas de TOC, com todas as suas obsessões e compulsões, vão além de alterações e transtornos mentais do indivíduo, interferindo também na sua qualidade de vida e de seus familiares e cuidadores. As consequências mais comuns do TOC são: diminuição da autoestima e do bem estar subjetivo, dificuldade na relação conjugal, interferência negativa na vida estudantil e profissional, dificuldades de relacionamentos, menor capacidade para apreciar atividades de lazer, além de custos indiretos para a família e a sociedade ${ }^{(6)}$. Em crianças e adolescentes acometidos por esse transtorno ocorre perda da capacidade de desempenhar as funções na escola, na sociedade e em casa, que pode estar relacionada à maior severidade da doença ${ }^{(7)}$.

O impacto negativo do TOC nos familiares é muito mais do que econômico, envolve as acomodações aos rituais e as solicitações bizarras do paciente, entre outros, motivando constantes conflitos, que pode levar a família a gerar sentimentos de compaixão, vergonha, raiva e muita ambivalência, cuja sobrecarga emocional pode ter efeitos deletérios na sua própria saúde mental ${ }^{(8)}$.

Subtipos específicos da doença obsessivo-compulsiva e seus diferentes níveis de gravidade podem sofrer influência de acordo com as maneiras da família lidar com o paciente, mas ainda não se pode definir se essas alterações familiares são causa ou consequência dos diferentes padrões de doença dos pacientes. Deve-se considerar também que alguns familiares tendem a ser portadores do transtorno, de forma plena ou subclínica, e que as influências biológica e ambiental constituem papel importante na manutenção dos sintomas ${ }^{(9)}$.

Embora ainda não definidas as verdadeiras causas do TOC, existem evidências de que diversos fatores de ordem biológica, envolvendo especialmente o funcionamento cerebral e fatores de ordem psicológica contribuam para $\mathrm{o}$ aparecimento e a manutenção dos sintomas ${ }^{(10)}$.

É importante considerar que as formas de tratamento, seja farmacológico ou comportamental, foram desenvolvidas e estão sendo utilizadas para melhorar a qualidade de vida do portador de TOC. Os medicamentos utilizados como antidepressivos a exemplo da clomipramina, paroxetina, fluoxetina, entre outros, também possuem ação antiobsessiva, geralmente com doses mais elevadas do que as utilizadas na depressão.

Anteriormente, até 1980, o atendimento e assistência ao portador de transtornos mentais acontecia somente nos hospitais psiquiátricos, se restringindo a internações e a tratamento medicamentoso apenas aos sintomas manifestados pelos doentes ${ }^{(11)}$. Nas três últimas décadas a introdução de métodos efetivos de tratamento, como a Terapia de Exposição e Prevenção de Respostas (EPR) e a Terapia Cognitivo-Comportamental (TCC), além do uso de medicamentos antiob- 
sessivos, contribuiu para mudar o antigo conceito de que o transtorno é de difícil tratamento ${ }^{(12)}$.

Diante desse quadro, o objetivo desde estudo foi caracterizar os pacientes acometidos por TOC, em uma instituição psiquiátrica da região Centro-Oeste de Minas Gerais.

\section{MÉTODO}

Trata-se de um estudo epidemiológico descritivo e retrospectivo realizado em uma instituição psiquiátrica da região Centro-Oeste do Estado de Minas Gerais, a qual presta assistência aos portadores de transtornos mentais de ambos os sexos da região, tanto para o atendimento de urgência, como ambulatorial e internação. A clínica na qual foi realizado o estudo é composta por equipe multiprofissional e possui estrutura física com capacidade de 120 leitos, 112 ativos, sendo $85 \%$ desses destinados ao Sistema Único de Saúde (SUS).

A coleta de dados foi realizada no Setor de Arquivo Médico e Estatístico, no qual o registro e o acompanhamento das internações estão armazenados sob a forma de prontuários. A clínica também possui um Sistema de Internação Hospitalar (SIH) eletrônico, contendo todas as informações acerca dos pacientes, o que também auxiliou a coleta e verificação dos dados.

Os dados coletados neste estudo foram submetidos ao Comitê de Ética em Pesquisa da Universidade de São Paulo/Campus de Ribeirão Preto, Protocolo n. 064912006. Foram adotados como critérios de inclusão: 1) pacientes internados no período de 20 de outubro de 1980 (data de inauguração da clínica) à 31 de dezembro de 2005; 2) com permanência superior a 24 horas; 3) que foram acompanhados desde a admissão, alta ou transferência. No total, foram utilizados 61 prontuários e todos atenderam aos critérios de inclusão, pois eram de pacientes portadores de TOC e as informações do banco de dados se encontravam completas. Deste modo, foram estabelecidas as seguintes variáveis: sexo, idade, procedência do paciente, tempo de internação, tipo de alta, tipo de internação e fonte financiadora. Os dados foram analisados pelo Software StatiscalPackage for Social Sciences (SPSS) versão 11.5.

\section{RESULTADOS}

Entre os 61 pacientes em estudo 14 (23\%) eram do sexo masculino e 47 (77\%) do feminino e foram admitidos na clínica no período de 20 de outubro de 1980 a 20 de outubro de 2005. Entre esses, 31 (50,8\%) eram provenientes da residência/família, 18 (29,5\%) de Centro de Atenção Psicossocial, 6 (9,8\%) de consultório médico particular e $6(9,8 \%)$ tinham outras procedências. Em relação à internação, $30(49,2 \%)$ caracterizava-se como primeira internação e $31(50,8 \%)$ como reinternação. As demais características estão apresentadas na tabela 1.

Tabela 1 - Caracterização dos pacientes admitidos na clínica portadores de Transtorno Obsessivo Compulsivo. Minas Gerais, 2011

\begin{tabular}{lc}
\hline Variável & $\mathbf{N}(\%)$ \\
\hline Idade & $1(1,6)$ \\
10 a 20 & $8(13,1)$ \\
21 a 30 & $7(11,4)$ \\
31 a 40 & $11(18,0)$ \\
41 a 50 & $10(16,3)$ \\
51 a 60 & $9(14,7)$ \\
61 a 70 & $11(18,0)$ \\
$>70$ & $4(6,5)$ \\
Ignorado & \\
Tempo de Internação & $1(1,6)$ \\
1 dia & $12(19,7)$ \\
2 a 10 dias & $5(8,2)$ \\
11 a 20 dias & $13(21,3)$ \\
21 a 30 dias & $28(45,9)$ \\
$>30$ dias & $2(3,3)$ \\
Outros & \\
Tipo de Alta Hospitalar & $51(83,6)$ \\
Alta Médica & $6(9,8)$ \\
Alta a Pedido & $2(3,3)$ \\
Alta Administrativa & $1(1,6)$ \\
Alta por Abandono/Evasão & $0(0,0)$ \\
Transferência Clínica & $0(0,0)$ \\
Óbito & $1(1,6)$ \\
Ignorado &
\end{tabular}

\section{Fonte Financiadora da Internação}

Sistema Único de Saúde $\quad 54(88,5)$

Particular $2(3,3)$

Convênio $2(3,3)$

Gratuito $1(1,6)$

Ignorado $2(3,3)$

Fonte: Banco de Dados do Sistema de Internação Hospitalar

\section{DISCUSSÃO}

Após a análise dos dados, observou-se que o TOC ocorre predominante no sexo feminino (77\%). Estes dados vão ao encontro da literatura ${ }^{(6)}$ que mostra que fatores como genética, influência exercida pelos hormônios sexuais femininos e a procura maior das 
mulheres por atendimento médico contribuem para essa predominância.

O TOC ainda se trata de transtorno subdiagnosticado e subtratado, muitas vezes secreto, pois portadores ocultam ao máximo seus sintomas e demoram muito tempo para procurar e obter tratamento adequado. Isto se deve, em grande parte, à habitual negação do portador em relação à doença e aos sintomas, que são acompanhados de vergonha, desmoralização e muito sofrimento, justamente pela noção preservada da irracionalidade de seus pensamentos e comportamentos e da incapacidade para mudá-los, além da consciência das próprias limitações ${ }^{(9)}$.

Quanto à faixa etária, as mais acometidas por TOC se encontram acima de 41 anos, com um total de 41 pacientes e uma frequência de $67 \%$. Pacientes idosos portadores de TOC são pouco citados na literatura ${ }^{(13)}$, o que é explicado pelo fato de há algum tempo atrás grande parte deles serem moradores da zona rural e possuírem características diferentes no processo de vida e envelhecimento quando comparadas às do meio urbano $^{(14)}$. Isto sugere que tinham um estilo de vida que os poupava do contato com o ritmo acelerado de vida, como o das grandes cidades, ou ainda, encontravam dificuldades em procurar serviços de saúde mental com o passar da idade, para os quais concorrem vários fatores, como crenças e atitudes dos idosos frente aos transtornos mentais ${ }^{(13)}$.

Outros fatores que podem explicar os poucos registros de pacientes idosos acometidos por TOC na literatura são a menor capacidade de os idosos perceberem a presença de transtornos mentais, sendo suas manifestações interpretadas como parte inevitável do envelhecimento, além da dificuldade de se estabelecer o diagnóstico em detrimento da existência de outros quadros patológicos, como a depressão e a demência, que são os transtornos mentais mais frequentes na terceira idade e também os transtornos ansiosos, o alcoolismo, os quadros maníacos, os transtornos mentais de origem orgânica, o uso abusivo e a dependência de sedativos. A prevalência de transtornos depressivos na população urbana idosa brasileira varia de 19,8 \% a $38,5 \%$. A demência apresenta frequências de $4,2 \%$ a 7,2\% em vários locais do Brasil e do mundo ${ }^{(15)}$.

Em relação à faixa etária de 41 a 50 anos, podemos destacar como fatores potenciais, para o desenvolvimento de algum transtorno psíquico, o estilo de vida urbano e muitas vezes agitado, além da crescente frustração com a rotina de trabalho. Esta frustração decorre da não adaptação dos indivíduos dessa faixa etária às atuais mudanças no mercado de trabalho. Antes eles eram acostumados com o modelo tradicional de trabalho, que não requeria qualificações e agora se veem competindo com jovens que estão mais adaptados à esta nova realidade e dominam as novas tecnologias ${ }^{(16)}$. O estudo da interação dos transtornos mentais e do comportamento relacionados ao trabalho tem obtido cada vez mais visibilidade nacional e internacionalmente. No Brasil, já foram atualizadas normas regulamentando o assunto, tais como: Nova Regulamentação sobre Doenças Profissionais e do Trabalho do Decreto n. 3048 do Ministério da Previdência e Assistência Social no dia 06/05/1999, e Lista de Doenças Relacionadas ao Trabalho da Portaria n. 1.339/GM, de 18/11/1999, que aborda também um capítulo específico sobre Transtornos Mentais e do Comportamento relacionados ao trabalho ${ }^{(17)}$.

Avaliada a proveniência, percebe-se que $31(50,8 \%)$ dos pacientes acometidos por TOC foram encaminhados ao tratamento pela própria família. Todavia vale ressaltar que a participação da família se distribui entre aquela que apoia e participa plenamente dos comportamentos (pólo de acomodação) até aquela que se recusa a participar e rejeita a doença (polo antagonístico). Há ainda um terceiro padrão em que a família se encontra dividida, com membros no polo de acomodação e outros membros no antagonístico ${ }^{(18)}$.

Quanto à variável tempo de internação, verificou-se que mais da metade dos pacientes $(54,1 \%)$ ficaram internados por períodos menores que 30 dias, fato justificado por alguns pacientes apresentarem resposta rápida, em poucas sessões de terapia ou com o uso dos medicamentos esses obtêm remissão completa dos sintomas $^{(12)}$. Existem também aqueles refratários a todas as abordagens, ou seja, não apresentam resposta aos tratamentos disponíveis, sejam os farmacológicos ou psicoterápicos, correspondendo à $45,9 \%$ que permaneceram internados por um período superior a 30 dias.

Quando analisado o tipo de alta hospitalar, verificou-se que $51(83,6 \%)$ dos pacientes recebem alta médica, seguido de alta a pedido com $6(9,8 \%)$ dos pacientes. Isso mostra boa adesão dos pacientes pelo tratamento, pois a maior parte deles recebem alta somente depois da autorização médica. Entretanto, é importante destacar que a melhora dos sintomas não reflete, necessariamente, em melhora funcional ou da qualidade de vida. É necessário acompanhamento do paciente para que dê continuidade ao tratamento a fim de obter o resultado esperado ${ }^{(8)}$.

Em relação à variável internação, houve paridade 
entre a proporção de pacientes que estavam em sua primeira internação $30(49,2 \%)$, e a de pacientes de reinternações 31 (50,8\%). Um percentual significativo de pacientes pode obter eliminação completa dos sintomas utilizando exclusivamente meios psicológicos, como a Terapia de Exposição e Prevenção de Respostas (EPR) ou a Terapia Cognitivo-comportamental (TCC) que, juntamente com os medicamentos antiobsessivos, constituem os tratamentos de primeira linha para o TOC. Entretanto, os dois grandes problemas enfrentados após a alta são: a grande dificuldade do paciente em dar continuidade ao tratamento terapêutico e ambulatorial (em razão do aumento da ansiedade que o tratamento pode provocar) e o abandono do tratamento (uma vez que o próprio paciente não acredita na sua capacidade de recuperação) ${ }^{(12)}$.

É fundamental esclarecer aos pacientes o que é TOC, quais as suas manifestações, quais os tratamentos disponíveis, além de outras dúvidas que possam surgir. Esclarecer essas questões e traçar estratégias de tratamento antes mesmo de iniciá-lo é essencial para que o paciente tenha uma boa adesão a ele, evitando fracassos e as consequentes reinternações ${ }^{(19)}$.

Ao se avaliar a fonte financiadora da internação, verificou-se que o SUS, foi responsável por 54 internações, o que corresponde a $88,5 \%$ do total. Como se trata de uma instituição filantrópica, na qual $80 \%$ dos leitos (96 de 120 ativos) são destinados ao SUS, era de se esperar tal prevalência. Porém, esse dado evidencia a continuidade da manutenção do sistema hospitalar para o tratamento dos transtornos psiquiátricos, pelo próprio SUS, contrariando a Lei n. 10.216 da reforma psiquiátrica. Ou seja, o próprio sistema público de saúde, que deveria ser o primeiro a fazer valer a lei, a ignora, promovendo internações com o dinheiro público.

Evidencia-se a necessidade de garantir aos portadores do TOC um acesso rápido e de qualidade a tratamentos adequados, principalmente nos serviços públicos de saúde. Deste modo, surge a necessidade de se tornarem mais efetivas as ações dos Centros de Atenção Psicossocial (CAPS), bem como promover a inserção e racionalização dos serviços de saúde mental na atenção básica, por intermédio da Estratégia de Saúde da Família, pois criam a possibilidade de estender a cobertura em saúde mental a um maior número de usuários. Devem ser desenvolvidas também estratégias de diagnóstico e tratamento precoces, bem como o acompanhamento dos pacientes com TOC em seu próprio cenário de vida, sem desvinculá-los de seu contexto social e prevenindo a sua segregação ${ }^{(20)}$.

\section{CONSIDERAÇÕES FINAIS}

Tomando como base os resultados encontrados, percebemos na população estudada que o TOC é uma patologia frequente em nossa sociedade e que fatores como ser do sexo feminino e possuir idade acima de 41 anos podem estar associados ao acometimento pela doença e a necessidade de tratamento.

Assim, percebemos que há necessidade de serem desenvolvidas ações que visem diagnosticar precocemente o transtorno, para que o tratamento seja iniciado o mais rápido possível a fim de se evitar o agravamento das obsessões e compulsões, o que pode resultar na necessidade de tratamento intensivo. Isto implica em uma maior capacitação e atualização dos profissionais de saúde, principalmente os que atuam na Atenção Primária (Saúde da Família), a fim de que revisem e atualizem continuamente as informações acerca do diagnóstico, etiologia e características principais da doença, o que torna o tratamento mais eficaz. Desse modo, haverá contribuição para a redução do estigma social gerado pela doença e do impacto econômico em todo o sistema de saúde.

Torna-se também investigar se os homens realmente são menos acometidos por TOC pois observamos na prática clínica que o menor registro de casos se deve à habitual negação ao adoecimento, associados à fragilidade, fraqueza e vergonha, o que resulta em uma menor procura por atendimento à saúde.

O maior contingente de pacientes acometidos por TOC foi encaminhado para o tratamento pela própria família. Neste sentido ressalta-se a importância de se oferecer atendimento às famílias, já que essas exercem papel fundamental na detecção dos sintomas, na promoção do tratamento e na cura dos pacientes. Deste modo, deve-se orientar as famílias quanto aos sintomas da doença, sobre a forma de atender as necessidades do portador do transtorno e a importância da continuidade do tratamento ambulatorial e domiciliar após a alta do paciente, pois sua melhora é gradativa. Deve-se promover o encorajamento do paciente por parte dessa família a fim de que o tratamento seja efetivo e não seja abandonado pelo doente.

No estudo foi perceptível a manutenção do sistema hospitalar para o tratamento psiquiátrico, assim como, pelos consultórios particulares e pelos CAPS que emitem encaminhamentos para a internação. Neste sentido, verificamos a necessidade de se fazer valer a Lei 10.216 da Reforma Psiquiátrica, por meio de investimentos na consolidação do Centro de Atenção Psicossocial como 
referência para a assistência em saúde mental.

\section{REFERÊNCIAS}

1. Paes F, Machado S, Velasques B, Ribeiro P, Nardi AE. Obsessive-compulsive disorder and eating disorders: a continuum or separate diagnoses? Rev. Bras. Psiquiatr. [Internet] 2011;33(2) [acesso em 04 set 2012]. Disponível: http://www.scielo.br/pdf/rbp/v33n2/ a20v33n2.pdf.

2. Soares Neto EB, Teles JBM, Rosa LCS. Sobrecarga em familiares de indivíduos com transtorno obsessivo-compulsivo. Rev. psiquiatr. clín. [Internet] 2011;38(2) [acesso em 04 set 2012]. Disponível: http://www.scielo.br/scielo.php? pid=S010160832011000200001\&script $=$ sci_arttext.

3. Mathis MA, Alvarenga P, Funaro G, Torresan R, Moraes I, Torres AR. Gender differences in obsessivecompulsive disorder: a literature review. Rev. Bras. Psiquiatr. [Internet] 2011;33(4) [acesso em 03 set 2012]. Disponível: http://www.scielo.br/scielo.php?pid=S151644462011000400014\&script=sci_arttext.

4. Pender AMG, Lambregtse-van den Berg MP, Raats ME. Obsessive compulsive disorder during pregnancy and the postpartum period. Tijdschr Psychiatr. [Internet] 2012;54(6) [acesso em 03 set 2012]. Disponível: http:// www.tijdschriftvoorpsychiatrie.nl/assets/articles/ TvP12-06-p549-553.pdf.

5. Nauta KJ, Batelaan NM, van Balkom AJ. Obsessivecompulsive disorder from a family perspective; implications for treatment and research. Tijdschr Psychiatr. [Internet] 2012;54(5) [acesso em 03 set 2012]. Disponível: http://www.tijdschriftvoorpsychiatrie.nl/ assets/articles/TvP12-05-p439-448.pdf.

6. Couto LSRB, Rodrigues L, Vivan AS, Kristensen $\mathrm{CH}$. A heterogeneidade do Transtorno ObsessivoCompulsivo (TOC): uma revisão seletiva da literatura. Contextos Clínic. [Internet] 2010;3(2) [acesso em 20 out 2011]. Disponível: http://pepsic. bvsalud.org/scielo.php?script=sci_pdf\&pid=S1983$34822010000200007 \& \operatorname{lng}=\mathrm{pt} \& \mathrm{nrm}=$ iso\&tlng $=\mathrm{pt}$

7. Bernard B. A review of obsessive-compulsive disorder in children and adolescents. Dialogues Clin Neurosci. [Internet] 2011;13(4) [acesso em 21 out 2011]. Disponível: http://www.ncbi.nlm.nih.gov/pmc/articles/ PMC3263388/.

8. Argimon IIL, Bicca MG, Rinaldi J. Transtorno obsessivo-compulsivo na adolescência. Rev. bras. ter. cogn. [Internet] 2007;3(1) [acesso em 02 fev 2012]. Disponível: http://rbtc.org.br/detalhe_artigo.asp?id=54.

9. Torresan RC, Smaira SI, Ramos-Cerqueira ATA, Torres AR. Qualidade de vida no transtorno obsessivo-compulsivo: uma revisão. Rev. psiq. clín. [Internet] 2008;35(1) [acesso em 24 out 2011]. Disponível: http://www.scielo.br/scielo.php?pid=S010160832010000300006\&script $=$ sci_arttext.

10. Stengler-Wenzke K, Kroll M, Matschinger H, Argermeyer MC. Subjective quality of life of patients with obsessive-compulsive disorder. Soc. Psychiatry Psychiatr Epidemiol. [Internet] 2008;41(8) [acesso em 25 out 2011]. Disponível: http://link.springer.com/ article/10.1007/s00127-006-0077-8.

11. Costa B, Inoue L, Kohiyama VY, Paiano M, Waidman MAP. Assistência de enfermagem domiciliar à família e portadores de transtorno mental: relato de experiência. Cogitare enferm. [Internet] 2010;15(2) [acesso em 24 ago 2012]. Disponível: http://ojs.c3sl.ufpr.br/ojs2/index. php/cogitare/article/view/17876/11666.

12. Cordiolli AV. A terapia cognitivo-comportamental no transtorno obsessivo-compulsivo. Rev. Bras. Psiquiatr. [Internet] 2008;30(2) [acesso em 26 out 2011]. Disponível: http://www.scielo.br/scielo.php?script=sci_ arttext\&pid=S1516-44462008000600003.

13. Pena MCS, Vale FAC. Obsessive-compulsive symptoms in dementia. Rev. psiq. clín. [Internet] 2010;37(3) [acesso em 03 nov 2012]. Disponível: http://www.scielo.br/scielo. php?pid=S0101-60832010000300006\&script=sci_arttext.

14. Morais EP, Rodrigues RAP, Gerhardt TE. Os idosos mais velhos no meio rural: realidade de vida e saúde de uma população do interior gaúcho. Texto Contexto Enferm. [Internet] 2008;17(2) [acesso em 03 nov 2012]. Disponível: http://www.scielo.br/pdf/tce/v17n2/21.pdf.

15. Lobosque AM. Debatendo alguns desafios da Reforma Psiquiátrica brasileira. Ciênc. saúde colet. [Internet] 2011;16(12) [acesso em 22 ago 2012]. Disponível: http://www.scielo.br/scielo.php?pid=S1413$81232011001300003 \&$ script $=$ sci arttext.

16. Kreling NH. A inserção do adulto maior de 40 anos no mercado de trabalho: ocupação e desemprego na RMPA. Indic. Econ. FEE. [Internet] 2004;31(4) [acesso em 02 nov 2012]. Disponível: http://revistas.fee.tche.br/index. php/indicadores/article/viewArticle/231.

17. Delgado PGG. Democracia e reforma psiquiátrica no Brasil. Ciênc. saúde colet. [Internet] 2011;16(12) [acesso 
em 23 ago 2012]. Disponível: http://www.scielo.br/ scielo.php?pid=S1413-81232011001300019\&script $=$ sci arttext.

18. Ferrão, YA, Florão, MS. Acomodação familiar e criticismo percebido em pacientes com transtorno obsessivo-compulsivo. J. Bras. Psiquiatr. [Internet] 2010;59(1) [acesso em 01 fev 2012]. Disponível: http://www.scielo.br/scielo.php?pid=S004720852010000100006\&script $=$ sci_abstract\&tlng=pt.

19. Clemente AS, Loyola Filho AI, Firmo JOA. Concepções sobre transtornos mentais e seu tratamento entre idosos atendidos em um serviço público de saúde mental. Cad. Saúde Pública. [Internet] 2011;27(3) [acesso em 25 ago 2012]. Disponível: http://www. scielo.br/scielo.php?script=sci_arttext\&pid=S0102311 X2011000300015.

20. Associação Brasileira de Psiquiatria. Transtorno Obsessivo Compulsivo: diagnóstico. Proj. Diretrizes. Associação Médica Brasileira e Agência Nacional de Saúde Suplementar. 2009. [acesso em 04 nov 2012]. Disponível: http://www.projetodiretrizes.org. br/ans/diretrizes/transtorno_obsessivo_compulsivodiagnostico.pdf. 